sich befanden, entdeckte, bewogen ihn, die akute Alkoholintoxikation genauer zu studieren. Von den Ergebnissen seiner diesbezüglichen, an 4 Studenten angestellten Untersuchungen wäre folgendes hervorzuheben: Ws traten spontaner Nystagmus geringen Grades, bei raschen Kopfbewegungen Nystagmusanfalle auf. Bei Neigung nach rechts war rotatorischer Nystagmus nach rechts, bei Neigung nach links rotatorischer Nystagmus nach links zu beobachten. Mit diesem $\mathrm{Ny}-$ stagmus war meist Schwindel geringen Grades verbunden. Die Dauer des Drehnystagmus wurde nicht verändert. Die Drehempfindung wurde wesentlich in ihrer Stärke herabgesetzt, ja bei einzelnen Kopfstellungen kam es sogar zu einer Aufhebung der Drehempfindung. Störungen der spontanen Zeigebewegungen wurden nicht beobachtet. Es trat spontanes Schwanken auf, unabhängig von dem eventuell bestehenden spontanen Nystagmus, meist auch unabhängig von der Kopfstellung. Die Reaktionsbewegungen der oberen Extremitäten wurden herabgesetzt und einzelne derselben vollkommen aufgehoben. Es traten abnorme Reaktionsbewegungen und abnorme Drehempfindungen auf, die nicht auf den gesetzten Reiz bezogen werden konnten. Stets gingen aber Reaktionsbewegungen und Drehempfindungen parallel. Aus den Versuchen scheint hervorzugehen, daß der Alkohol bei der akuten Alkoholvergiftung eine lähmende Wirkung auf das Kleinhirn ausuibt, die man durch Prüfung der vestibularen Reaktionen objektiv konstatieren kann. Vortr. sucht eine Erklärung für diese Erscheinungen in jenen Bahnen, die vom Kleinhirn über den roten Kern zur Großhirnrinde ziehen.

\title{
Disk assion.
}

Neumann: Wenn ich Herrn Bá rány richtig verstanden habe, so nimmt er an, daß die Drehempfindung und der Zeigeversuch auf denselben Bahnen dem Kleinhirn zugeleitet werden. Ich möchte mir dann nur die Anfrage gestatten, ob er sich erinnern kann, dah ich hier einen Fall demonstriert habe, bei dem im peripheren Gehörorgan nichts Abnormes zu konstatieren wax, indem Patient sowohl für die Stimmgabel als auch für die Sprache ein normales Gehör aufwies, der Vestibularapparat für alle Reize prompt erregbar war, und doch fehlte bei demselben die Drehnachempfindung bei Vorhandensein eines normalen Zeigeversuches.

$$
\text { H. Ne u } \mathbf{m} \text { a } \mathbf{n}-\text { Wien. }
$$

\section{Berliner Otologische Gesellschaft.}

\author{
Sitzung vom 31 . M ärz 1911 .
}

1. Herr Halle: Myalgien in der Ohrenheil$\mathrm{k}$ und e. (Über die ausführlich in der M. f. $\mathrm{O}$. erschienene Arbeit wird später berichtet werden.)

\section{Dis kus sion.}

Herr $\mathrm{R}$ it t e r und $\mathrm{Cl}$ a $\mathrm{us}$ glauben nicht, daß es sich um ein neues Krankheitsbild handelt.

Herr $\mathrm{P}$ exit $\%$ (a. $\mathrm{G}$.) empfiehlt in jede einzelne schmerzempfindliche Stelle Injektionen einer Lösung von 0,6 Chlornatrium und 0,075 Kalzium in $100 \mathrm{~g}$ Wasser.

Herr $\mathrm{H}$ a e $\mathrm{n} l$ e i n empfiehlt Nervenmassage.

Herr G r o B m a n schlägt einen Versuch mit Hypnose vor.

2. Herr Wolff: Untersuchungen mit dem Monochord. Dasselbe gibt vollständigere Resultate als die Pru- 
fung mit Pfeifen, da die Monochordtöne auch auf dem. Knochenwege zugeleitet werden können. Redner bespricht die Fortpflanzungsart von Stimmgabel- und Monochordtönen durch die Kopflknochen. Auch Transversaltöne des Monochords $\left(\mathrm{g}^{1}-\mathrm{g}^{4}\right)$ wurden angewandt. Resultat: Bei nervöser Schwerhörigkeit ist die Knochenleitung für tiefe, die Luftleitung für die höchsten Töne verschlechtert. Das Optimum der Tonzuleitung für tiefe Töne ist die Luftleitung, für die höchsten Töne die Knochenleitung.

Sitzung vom 19. Mai 1911 .

Krankendemonstrationen.

Herr Levy: AufmeiBelang wegen $K a r i e s$ bis z um Jochbogen.

Herr Pass ow: Postdiphtheritische Verwachsung des weichen Gaumens mit der $R$ achenwand, mit dem geknöpften Messer getrennt und durch eine Prothese am Wiederverwachsen gehindert.

Herr Graupner: 2 Mädchen mit eigentümli che m Trommelfellbefund bei Lues hereditaria. Die Veränderungen am Hammergriff hält $G$. für Periostitis gummosa.

1. Herr Kalähnè: Untersuchungen über die Wahrnehmung höchster Töne durch Luft-und Knochenleitung mittels des Monochords.

Die höchsten Töne sind weiter hinauf wahrzunehmen durch die Knochenleitung als durch Luftleitung.

Bei Ohrkranken ist die Knochenleitungsgrenze immer höher als die Luftleitungsgrenze gelegen.

Die Luftleitung geht von 15000 bis 18000 , die Knochenleitung von 17000 bis 21000 Doppelschwingungen.

Bei Mittelohrerkrankungen war die Luftleitungsgrenze stets stärker exniedrigt als die Knochenleitungsgrenze.

Bei Otosklerose ist die Knochenleitung anfangs lange normal bei gleichzeitig starker Erniedrigung der Luftleitungsgrenze.

Bei Unfallgutachten soll man aus einer kleinen Herabsetzung der oberen Tongrenze für Luftleitung keine falschen Schlüsse ziehen, da bei Otitis media die Luftleitungsgremze oft erniedrigt ist und manchmal bleibt.

\section{Disk us ion.}

Herr Wolff fand, wie $H$ el $\mathrm{m}$ holtz und $\mathrm{H}$ ege ner, als höchste Grenze 23000 Doppelschwingungen.

Herr $\mathrm{K}$ a $1 \ddot{a} \mathrm{~h} n$ e bemerkt, daß schon bei leichtem Tubenkatarrh manchmal eine geringe Einschränkung der oberen Tongrenze besteht.

\section{Sitzung vom 23 . Juni 1911 . \\ Demonstrationen.}

Herr $\mathrm{K}$ r $\mathrm{m}$ b e in: Fall von allmählich zunehmender Schwerhörigkeit beiderseits nach einem $R$ evolverschuB in den $\mathrm{K}$ o p f durch eine starke narbige Verengerung der Tuba, die keine Bougierung ermöglicht. Leichte linksseitige Facialisparese. Kreuzförmige strahlige Narbe am Rachendach.

Dis kus ion: Herr Gutzman , K r u m ein.

Herr $H$ erzfeld demonstriert einen $A$ p par a $\mathrm{z}$ ur $V$ ornahmeder kalorischen Reaktion mittels kalter Lift.

Diskussion: Herr Lautenschläger benutzt für die gleiche Prüfung einen komplizierten elektrischen Apparat. 


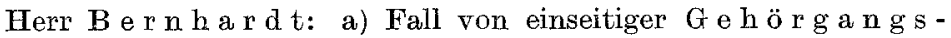
striktur nach chronischer Eiterung. Durchmesser $2 \mathrm{~mm}$. b) Fall von $\mathrm{I}$ u et is cher E $\mathrm{r}$ t a $\mathrm{u}$ b u $\mathrm{ng}$ auf beiden Ohren; nach intravenöser Injektion von $0,6 \mathrm{~g}$ Salvarsan trat sofort erhebliche Besserung ein.

1. Herr Krumbein: Otitis luetica. Bei 4 Fällen von hereditärer Syphilis war zweimal der Vestibularapparat ausgeschaltet neben vollkommener Taubheit, einmal war die statische Funktion vermindert neben Schwerhörigkeit, einmal fand sich beiderseits ein normal funktionierender Vestibularapparat neben labyrinthärer Schwerhörigkeit der einen Seite. Bei der erworbenen Lues (3 Fälle) war, in Ưbereinstimmung mit den Angaben von $\mathbf{S}$ i e b e $n$ ma $n n$, die Erregbarkeit des Vestibularapparates erhalten, bzw. gesteigert. 6 Fälle wurden mit Salvarsan behandelt, davon zeigten 3 Besserung des Sprachgehörs, 1 Besserung des Tongehörs. Ein Fall zeigte etwa 6 Wochen nach der Hatainjektion neben Besserung des Gehörs einen vollkommenen Ausfall der kalorischen und eine Herabsetzung der Drehreaktion. Vortr. faßt dieses entweder als $\mathrm{H}$ e $\mathbf{r} \mathbf{x} \mathbf{h}$ e ime rsche Reaktion oder als Ausdruck einer spezifischen Wirkung des Salvarsans auf den Vestibularis auf.

\section{Dis k us sion.}

Herr $\mathrm{H}$ erzfeld hält die erwähnte Reaktion nicht für eine Her $\mathrm{xh}$ i m e r sche, da sie erst nach 6 Wochen erschienen ist. Auch dem Salvarsan gibt er keine Schuld für die Störungen am Vestibularapparat; ex hat bei zwei mit Quecksilber behandelten und bei einem überhaupt nicht behandelten Luetiker das gleiche gesehen.

Herr S c h w a b a c h schließt sich den von H e r z f eld gemachten Ausführungen an.

Herr $\mathrm{L}$ e $\mathrm{v}$ y macht einen Unterschied in bezug auf Erscheinungen, die nach 8 Tagen, und solche, die nach 6 Wochen auftreten. Ihm ist es zweifelhaft, ob es sich bei den sich nach 5- 6 Wochen zeigenden um ein Neurorezidiv oder um Salvarsanwirkung handelt. Der von ihmo vor einigen Sitzungen erwähnte Patient ist nach zwei weiteren Salvarsandosen von $0,2 \mathrm{~g}$ auf dem einen Ohre taub geblieben, auf dem anderen ist eine Besserung eingetreten.

Herr $\mathrm{Krumb}$ i $\mathrm{m}$ betont zum Schlusse nochmals, daß seiner Meinung nach das Salvarsan die Funktion des Cochlearis bessert, dagegen diejenige des Vestibularis schlecht beeinflußt.

2. Herr Meyer: UUber plastischen VerschluB persistenter ơffnungen nach Kieferhöhlenoperation en. M.s Verfahren stellt einen VerschluB in zwei Etagen dar.

In der $\mathrm{D}$ is $\mathrm{k}$ us i o $\mathrm{n}$ bemerken die Herren C I a s, L a u tenschläger und $\mathrm{P}$ ass ow, daß sie ein gleiches Verfahren schon lange angewendet haben.

$\mathrm{W}$ i n d s c h ü g l - Charlottenburg.

\section{Belgische Gesellschaft für Otologie, Laryngologie und Rhinologie.}

XXI. Jahresversamm I ung in Gent am $15 ., 16$. und 17. Juli 1911 .

Vorsitzender: J. B r o e c k a e rt (Gent).

A. Demonstration anatomiseher Präparate.

1. Bôval (Charleroi): Zahnhaitige Zyste in der $\mathrm{K}$ i ef e $\mathrm{r} h$ öhle. Tumor vom Umfang einer großen $\mathrm{NuB}$, in seinem 